\title{
PERFORMANCE EVALUATION OF MOBILITY AND ROUTING PROTOCOLS FOR VEHICULAR AD HOC NETWORKS USING NS-2 AND VANETMOBISIM
}

\author{
Fatma Baccar $^{1}$, Kais Mnif ${ }^{1}$ and Lotfi Kammoun ${ }^{2}$ \\ ${ }^{1}$ National School of Electronics and Telecommunications, \\ NTS'Com, Sfax, Tunisia \\ ${ }^{2}$ National School Engineers of Sfax, Sfax, Tunisia
}

\begin{abstract}
In this paper, we will focus on the performance evaluation of a vehicular mobility scenario graph. Indeed, we will analyze the performance metrics (throughput, packets loss and end to end delay) using the IEEE 802.11 p standard of the proposed mobility graph. In addition, we examine the impact of the packet length, vehicle speed and routing protocols (Ad-Hoc Ondemand Distance Vector "AODV", Destination Sequenced Distance Vector "DSDV" and DumbAgent). Our simulations are based on the networks simulator "NS-2" and the mobility simulator "VanetMobisim" to evaluate the performance of the vehicular ad hoc network.
\end{abstract}

\section{KEYWORDS}

Vanet; mobility graph; 802.11p; throughput; end to end delay; packets loss; routing protocols; vehicle speed; packet size; NS-2; VanetMobisim

\section{INTRODUCTION}

Vehicular Ad hoc Networks (VANETs) are a special type of Ad Hoc networks; it is a subclass of mobile Ad hoc networks (MANETs). The purpose of this network is to provide safety, to minimize road accident and to give new interests for inter vehicle communications by: helping emergency vehicles to pass other vehicles quickly, broadcasting warning messages to neighboring vehicles in case of car accidents, providing drivers with latest real time traffic information, assisting drivers to find accessible parking space [1], etc. VANET is different from other kinds of networks; some of its characteristics are: high mobility, dynamic topology, short communication periods and limited bandwidth. VANET communications are based on broadcast messages which are exchanged between vehicles. The IEEE $802.11 \mathrm{p}$ is employed as a transmission protocol. It's an approved extension to the IEEE 802.11 standard, adding wireless access in vehicular environments (WAVE), for easier and more effective communication between vehicles with dynamic mobility. This technology uses the $5.9 \mathrm{GHz}$ band in different propagation environments [2].

The simulation was used to evaluate the performance of VANET due to the high cost of deploying and implementing VANET systems in a real environment. A lot of simulators for VANETs have been emerging [3] [4]. One of the first attempts to authors was to present a comprehensive study and to make comparison of the various publicly available VANET simulation software and their components [5] [6] [7].

Natarajan Meghanathan et al. (Eds) : NeTCoM, CSEIT, GRAPH-HOC, NCS, SIPR - 2017

pp. 71-83, 2017. (C) CS \& IT-CSCP 2017

DOI : $10.5121 /$ csit.2017.71507 
After study, we have decided to work with VanetMobisim simulator [8] which can be used to understand the properties of the mobility graphs of vehicular traffic and to interface it with NS-2 [9] in order to evaluate the performance metrics using the parameters of 802.11p standard. The traffic simulator VanetMobisim is an open source and it supports Intelligent Driver graph with Intersection Management (IDM_IM) which generates realistic vehicular mobility graph [10].

Several publications [11] [12] [13] have studied the performance for vehicular ad hoc networks; however they do not support realistic vehicular mobility simulation. Mostly, the authors simulate the mobility and the routing protocol separately.

The rest of the paper is structured as follows. In section 2, we will briefly describe DumbAgent, AODV and DSDV protocols. In section 3, we will describe the IDM_IM mobility model graph<. The simulation system structure, description of the scenario graph and simulation results will be discussed in section 4. Finally, the conclusions and future work are presented in Section 5.

\section{ROUTING PROTOCOL}

For our performance comparison study, we choose the most known and the most used routing protocols for VANET such as DumbAgent, AODV and DSDV were chosen. We will shortly describe these protocols in the following section.

\subsection{DumbAgent protocol}

DumbAgent routing protocol host agents and give a place to store a database of routing information. The technique is to embody the intelligence in the system. When vehicle moves from node to node, it updates its routing information as they go. The goal of the routing agent is to explore the network and to update every visited node. Each routing agent memorizes a history of where it has been. At each node, the agent uses its information history to update its routing table with adding the best paths.

\subsection{DSDV protocol}

Destination sequenced distance vector protocol (DSDV) [14] is a proactive routing protocol which is an amelioration of the Distributed Bellman Ford algorithm. DSDV tries to solve the routing loop problem. It provides one path only between source and destination, which is computed using the distance vector algorithm. To reduce the network overhead, two types of update packets are used: full dump and incremental update.

\subsection{AODV protocol}

Ad-Hoc On-demand Distance Vector protocol (AODV) [15] [16] is a reactive routing protocol which is based on DSDV protocol, therefore, paths are determined by sources only when needed to reduce traffic overhead. Paths are maintained only as long as data are traveling along the routes from the sender to the receiver. AODV protocol provides unicast and multicast communication. In this protocol, the sequence numbers are used for loop prevention and as route freshness criteria.

\section{MOBILITY MODEL GRAPH}

In order to model a realistic vehicular movement in our simulation, it's preferable to use a realistic mobility model graph. So, we use the Intelligent Driver Model with Intersection Management (IDM_IM) [10]. Generally, it's the most mobility model graph used and it's a part 
of the VanetMobisim tools. The Intelligent Driver Model (IDM) is a macroscopic car following model that adapts automatically a vehicle speed according to other vehicles driving ahead. This type is one of the cars following models category [3]. IDM_IM model uses a small set of parameters, which can be calculating with the help of real traffic measurements. This model is an extension of the IDM model, in order to introduce the management of intersections regulated by traffic lights and of broads with multiple lanes [17]. It can manage crossroads regulated by both stop signs and traffic lights.

\section{Performance Evaluation}

\subsection{Simulation System Structure}

Our simulation process is shown in figure 1. First; we use the VanetMobisim to describe the scenario by defining the environment details and the vehicle mobility using XML language. After running VanetMobisim simulator, it generates a trace file that contains the vehicular traffic and all environment details (node identifier, position, simulation time, vehicular speed...). Next, the Network Simulator NS-2 takes as input the generated file of VanetMobisim. NS-2 uses the Tcl script as a programming language to describe the details related to communications and network configuration. Finally, we get two files (*.nam and *.tr) as the outputs after running NS-2. The Network Animator file records all the positioning and graphical informations performed during the simulation time (see figure 2) of the scenario. The trace file contains all of the informations about the simulation results (packets sent, received and dropped, attached sequence number, protocol type, packet sizes...) To extract the statistics of the performance metrics from the generated file of NS2, we use AWK tool.

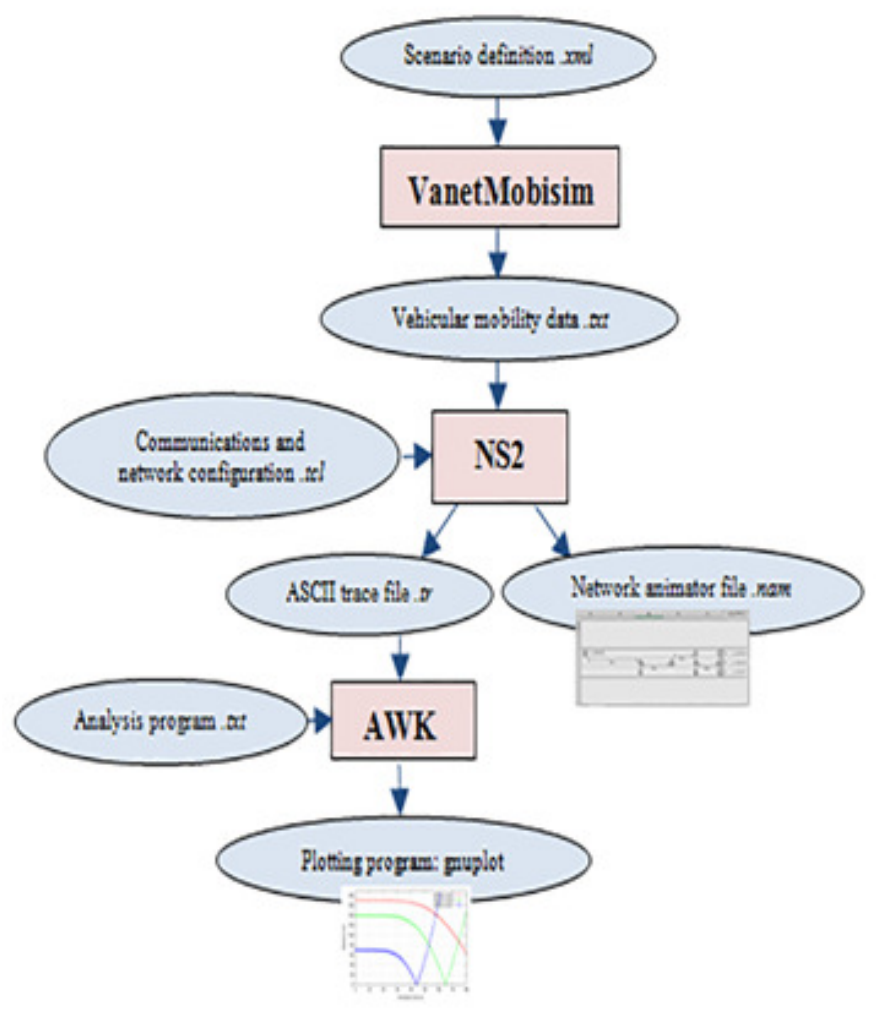

Figure 1. Simulation system structure 


\subsection{Scenario}

Our test scenario aims to present a real situation. The scenario represents a part of a highway. This part of the road comprises ten vehicles dispersed on three parallel unidirectional lanes (see figure 2). The cars have a minimum speed equal to $60 \mathrm{~km} / \mathrm{h}$ and a maximum speed around 70,100 and $120 \mathrm{~km} / \mathrm{h}$ respectively in lanes 1,2 and 3 . In exception, the vehicle 0 is a police car and it has a maximum speed equal to $140 \mathrm{~km} / \mathrm{h}$, the police car is in the emergency situation and diffuses a periodic message every 0.2 seconds with a payload equal to 500 bytes. To better evaluate the performance metrics, we vary the size of the packets sent by the police vehicle as well as the routing protocol used (DumbAgent, AODV and DSDV). Tab.I resumes all simulation parameters.

Table1. Simulation parameters

\begin{tabular}{|l|l|}
\hline Simulation environment & Ubuntu 14.04 \\
\hline Traffic simulator & VanetMobisim \\
\hline Network simulator & NS2 \\
\hline Simulation time & 80 seconds \\
\hline Simulation area & $2000 * 300$ meters \\
\hline Number of nodes & 10 \\
\hline Mobility model & IDM_IM \\
\hline Packet size & $500,1000,1500$ bytes \\
\hline \multirow{3}{*}{ Max speed } & Lane $1: 70 \mathrm{~km} / \mathrm{h}$ \\
\cline { 2 - 2 } & Lane $2: 100 \mathrm{~km} / \mathrm{h}$ \\
\cline { 2 - 2 } Lane $3: 120 \mathrm{~km} / \mathrm{h}$ \\
\hline MAC protocol & $802.11 \mathrm{p}$ \\
\hline Radio propagation model & Two Ray Ground \\
\hline \multirow{2}{*}{ Routing protocol } & $\begin{array}{l}\text { DumbAgent, } \\
\text { DSDV }\end{array}$ \\
\hline
\end{tabular}

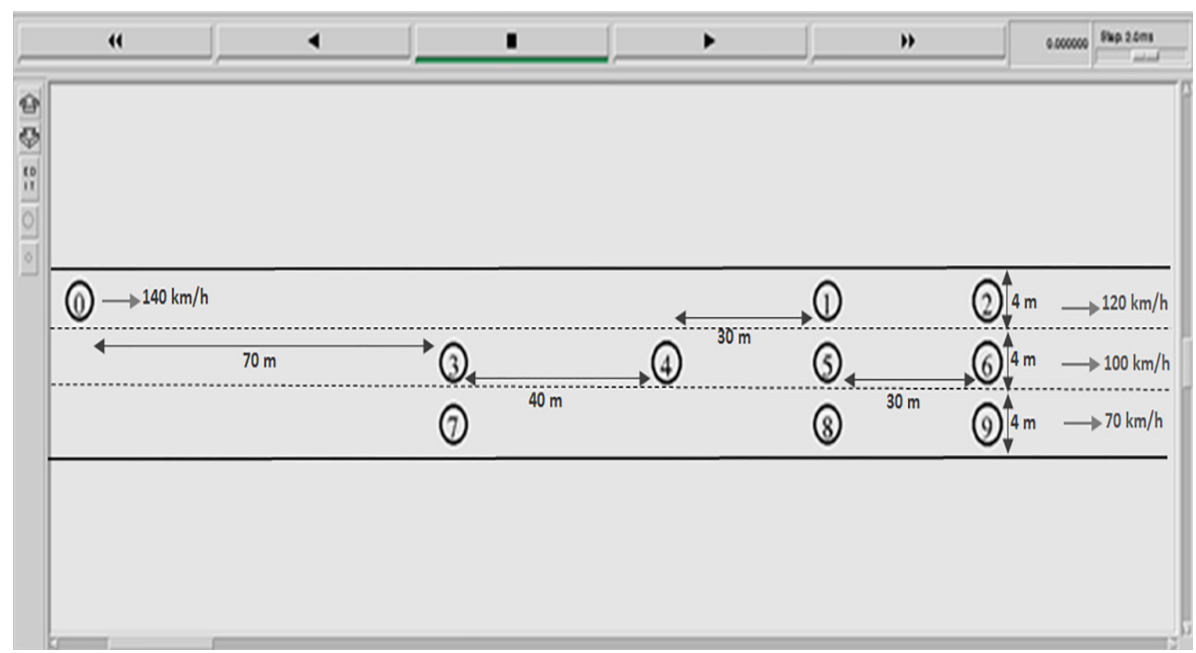

Figure 2. Simulation scenario graph 


\subsection{Results}

In this subsection, we will present the simulation results obtained of the scenario described in figure 2 .

We consider the throughput, the end to end delay and the packet loss of the nine vehicles when the vehicle 0 transmits a periodic packet with a payload equal to 500 bytes. The protocol used in this case is the DumbAgent for more evaluating the performance metrics with the $802.11 \mathrm{p}$ standard.

\section{- Throughput}

$T$ is the throughput : it is the rate of successful packet delivery through a network connection per unit of time:

$$
T=\frac{S(t)}{t}
$$

Where, $t$ is the unit of time taken and $S(t)$ is the total successful packet received at the defined time $t$.

\section{- $\quad$ End to end delay}

$D_{i}$ is the end to end delay. This term includes all the time required for a packet to be generated, transmitted through the network, and received by the destination:

$$
D_{i}=t r_{i}-t s_{i}
$$

Where, $i$ is the packet identifier, $t r_{i}$ is the time at which a packet is received at destination, $t s_{i}$ is the time at which a packet is sent from source.

$D_{\text {average }}$ is the average end to end delay:

$$
D_{\text {average }}=\frac{1}{n} \sum_{i=1}^{n}\left(t r_{i}-t s_{i}\right)
$$

Where, $n$ is the number of packets which delivered successfully.

\section{- Packet loss}

The reliability of the network connection is evaluated by the packet loss rate $P_{\text {loss }}$.

$$
P_{\text {loss }}=\frac{L}{p}
$$

Where, $L$ is the number of non received packets and $p$ is the total number of sent packets.

Figure 3 illustrates the distances separating the police car and other vehicles 2, 5 and 7 during the simulation time. 


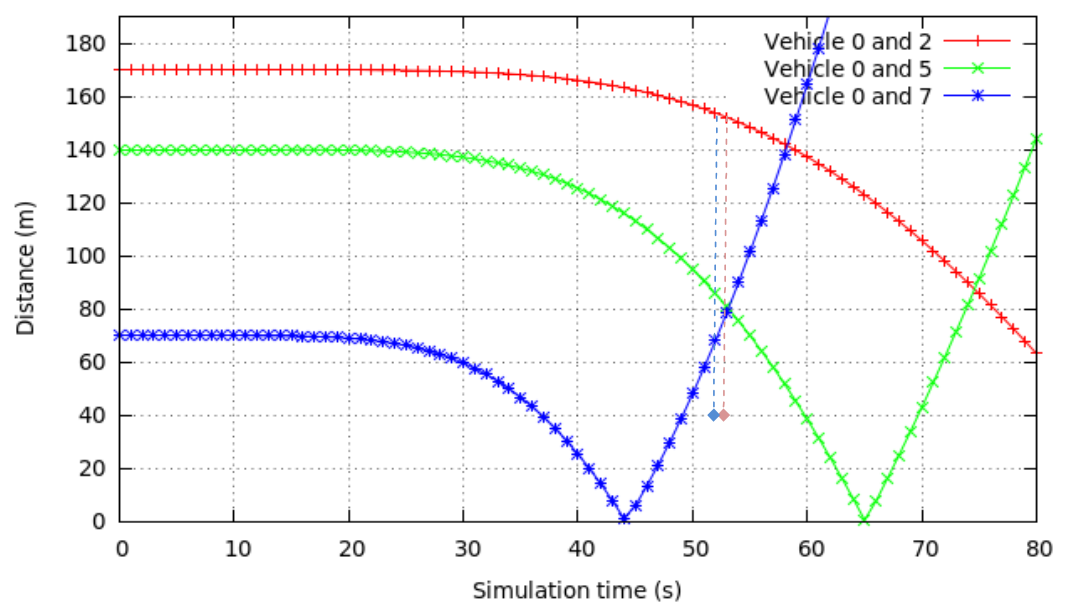

Figure 3. Distance between vehicle 0 and vehicles 2, 5 and 7

The throughput of vehicle 2, 5 and 7 with a packet size 500 bytes is shown in figure 4 . We can notice that the throughput of vehicle 2 is equal to $0 \mathrm{kbps}$ for 59 seconds but after this time it becomes between 3.6 and $4.4 \mathrm{kbps}$. According to figure 3, the distance between the police car and vehicle 2 is greater than 140 meters for 59 seconds. The time of state transformation of the throughput is 58 seconds for the vehicle 7. The throughput of vehicle 5 does not reach $0 \mathrm{kbps}$; this is due to the distance that separates it from the vehicle 0 which still less than 140 meters according to figure 3 .

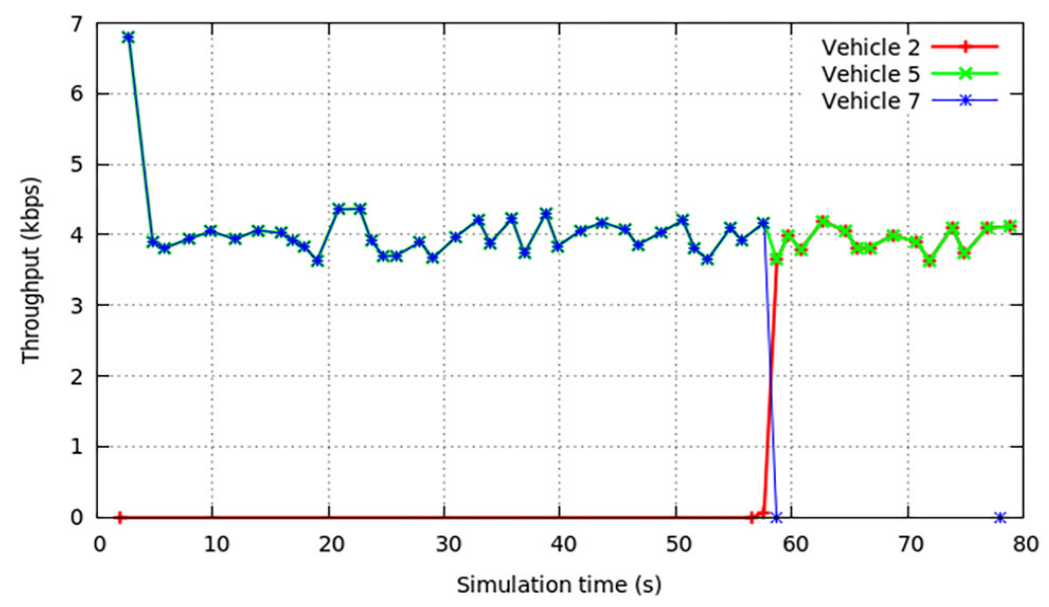

Figure 4. Throughput of vehicles 2, 5 and 7 (packet size: 500 bytes)

Figure 5 shows the percentage of packet loss between vehicle 0 and vehicles 2,5 and 7 during simulation time. The packet loss between police car and vehicle 2 and vehicle 7 is $0 \%$ when the distance between the vehicle 0 and the other vehicles is less than 140 meters. All the simulation time, there is no packet loss between vehicle 0 and vehicle 5 .

We can conclude that all vehicles with a distance less than 140 meters from vehicle 0 have the same throughput and have no packets loss with the police car. 


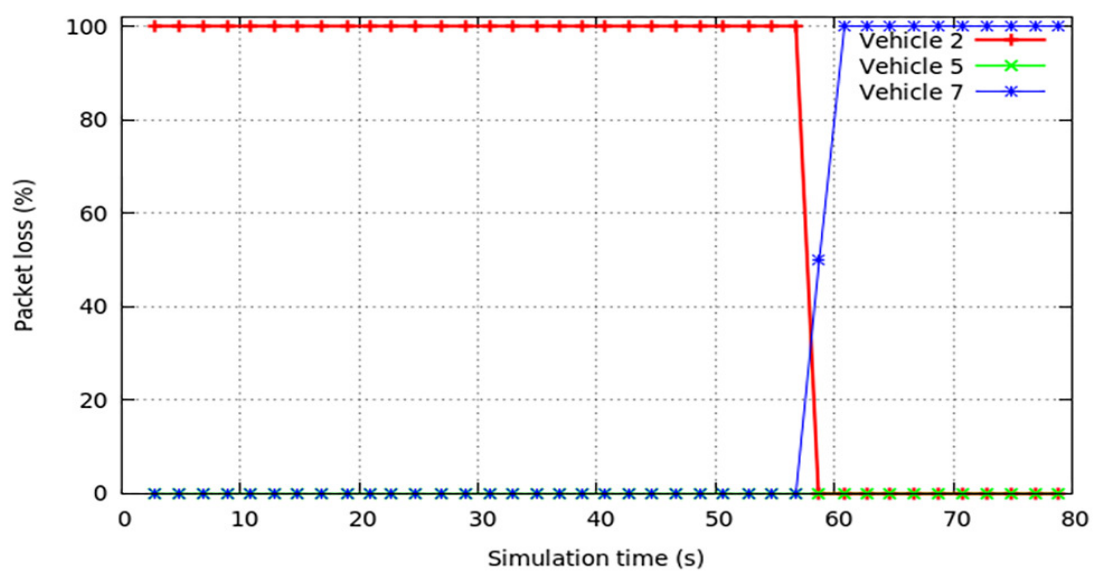

Figure 5. Packet loss between vehicle 0 and vehicles 2, 5 and 7 (packet size : 500 bytes)

Figure 6 presents the end to end delay between police car and vehicle 2, 5 and 7 . We may notice that when the distance between vehicle 0 and other vehicles is less than 140 meters, we have a tracing of end to end delay. According to figure 3, when the distance between the transmitter and the receiver decreases, the end to end delay decreases as well.

So, as a result the throughput, the packet loss and the end to end delay are affected by varying the distance between police car and other vehicles and not by varying the speed of vehicles.

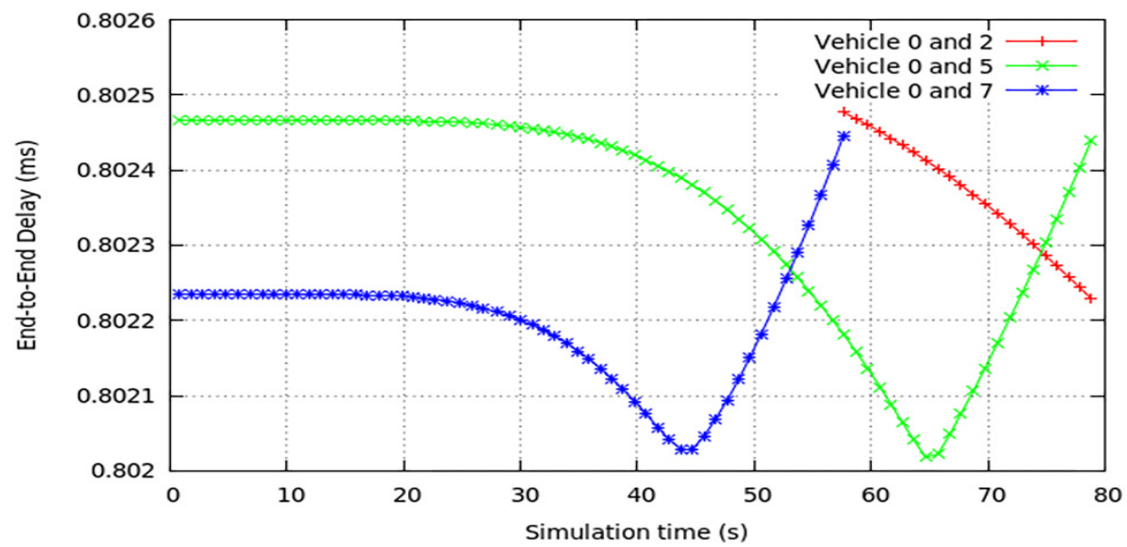

Figure 6. End to end delay between vehicle 0 and vehicles 2, 5 and 7

(packet size : 500 bytes)

To obtain a statistical significance, we calculate the average performance metrics in figure 7 , figure 8 and figure 9 .

Figure 7 illustrates the average throughput off all vehicles with packet size 500 bytes. We can show that the vehicles 2, 6 and 9 have the lowest value of throughput. The average throughput of others vehicles can reach 4 kbps. 


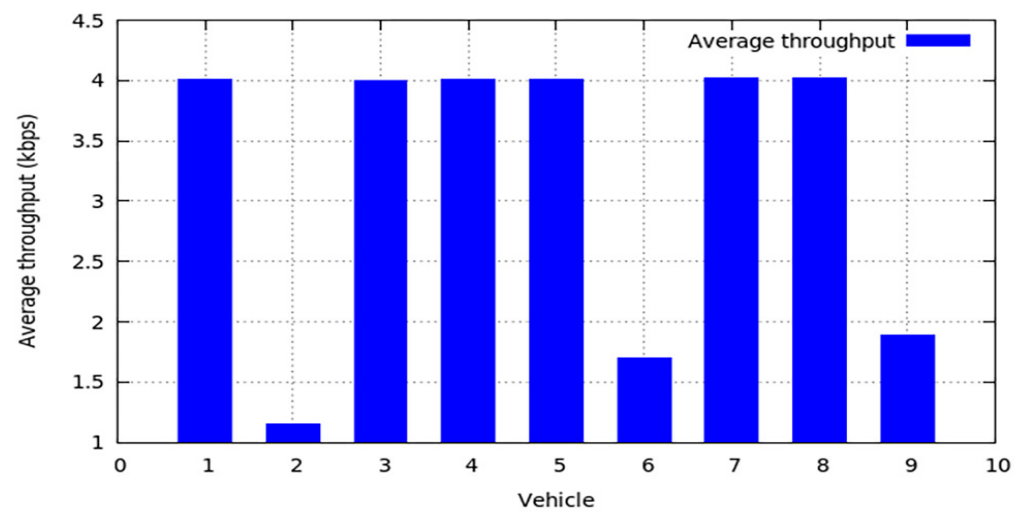

Figure 7. Average throughput of all vehicles (packet size: 500 bytes)

The percentage of the average packet loss is present in figure 8. Since the vehicles 2, 6 and 9 have had the minimum value of average throughput in figure 7, they have the maximum value of average packet loss between them and vehicle 0 . As a result, the vehicle 1 and 5 haven't lost any packet with the police car.

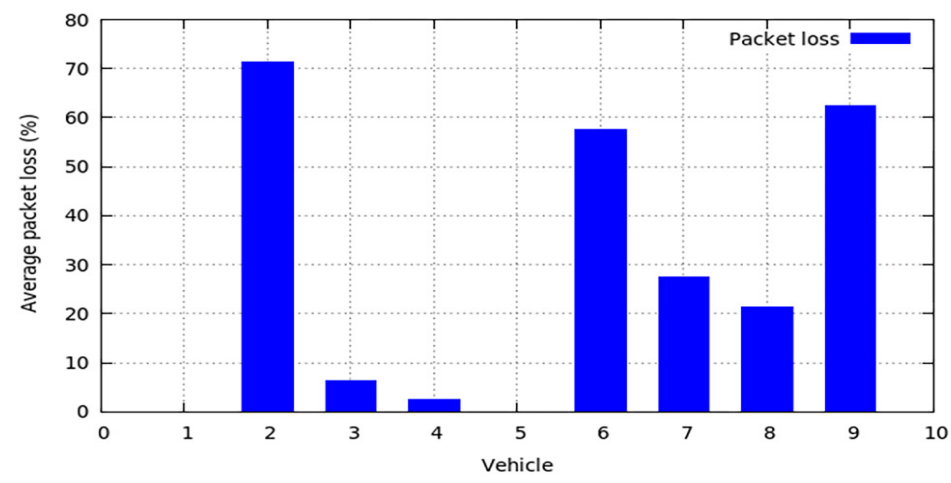

Figure 8. Average packet loss off all vehicles (packet size : 500 bytes)

The average end to end delay of all vehicles is calculated in figure 9 with packet size 500 bytes. The average value of the end to end delay between transmitter and receiver is almost the same for all vehicles $(0.8 \mathrm{~ms})$.

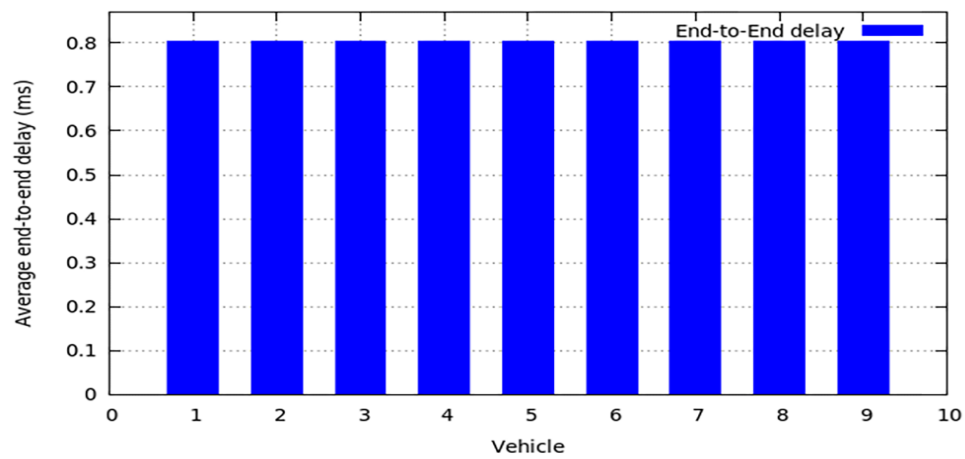

Figure 9. Average of the end to end delay between vehicle 0 and other vehicles (packet size : 500 bytes) 
In figure 10 and figure 11 , we modified the packet size sent by the police car to evaluate better the effect of the packet size in the performance metrics. The vehicle 0 sent a periodic packet every 0.2 seconds with a payload size of 500,1000 and 1500 bytes respectively in three network simulations. Figure 10 and figure 11 show the average throughput and end to end delay of all vehicles with three different packet sizes. As the packet size increases, the average throughput and the average end to end delay increases as well, but the increment of throughput of vehicles 2 , 6 and 9 is not as high as other vehicles.

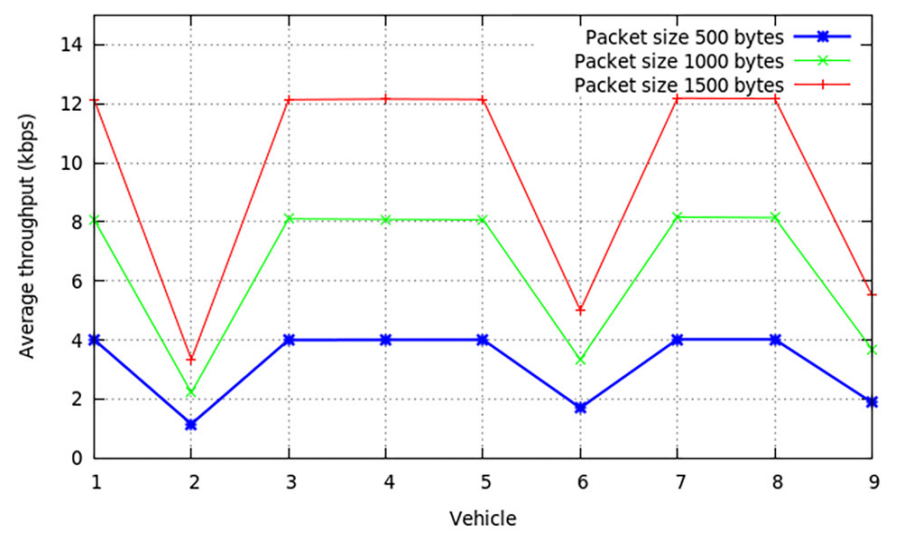

Figure 10. Average throughput of all vehicles (packet size : 500, 1000, 1500 bytes)

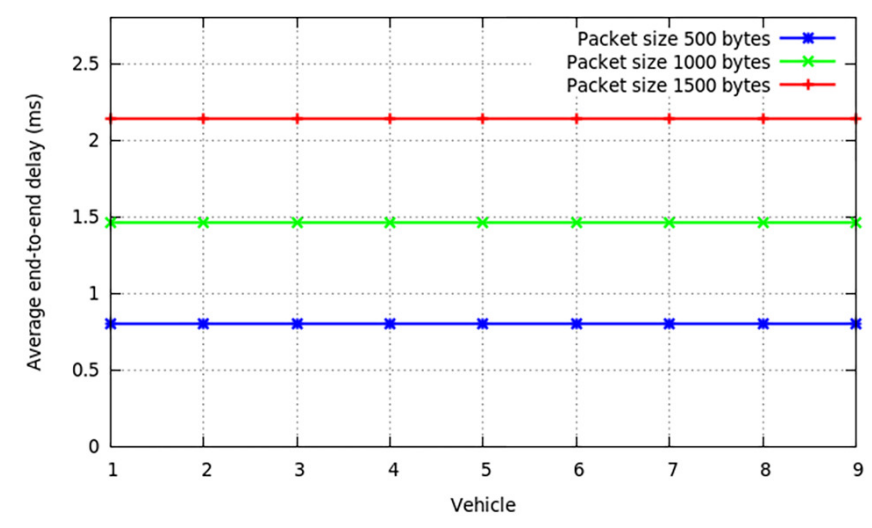

Figure 11. Average end to end delay between vehicle 0 and other vehicles (packet size : 500, 100, 1500 bytes)

In the end of this paper, we will evaluate the effect of different routing protocols (DumbAgent, AODV and DSDV) on the performance metrics. For example, we choose in simulation the vehicle 7 with a packet size 500 bytes.

Figure 12 illustrates the throughput of vehicle 7 and figure 13 shows the packet loss between vehicle 0 and vehicle 7 with different protocols. We note that the different protocols give almost the same results in the two figures. 


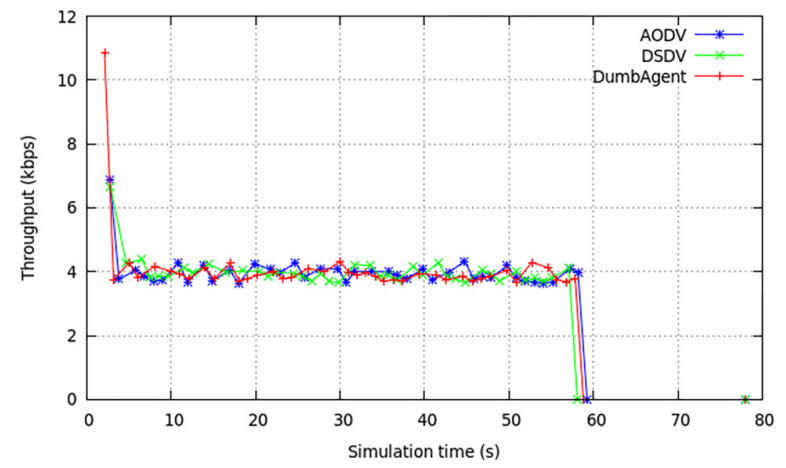

Figure 12. Throughput of vehicle 7 with different protocols (packet size: 500 bytes)

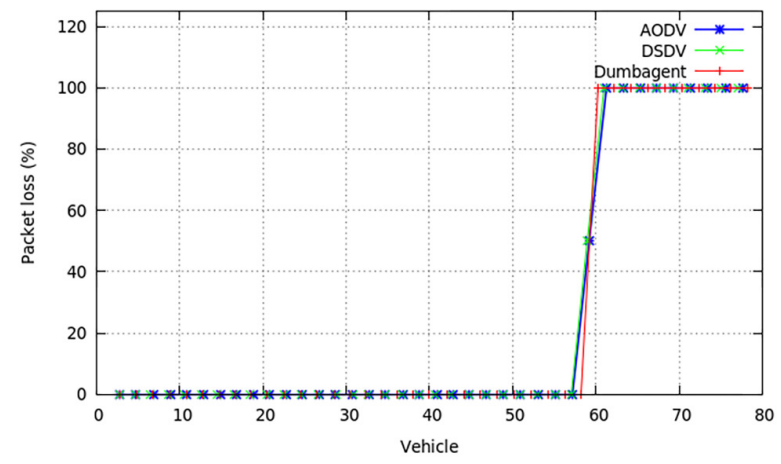

Figure 13. Packet loss between vehicle 0 and vehicle 7 with different protocols (packet size : 500 bytes)

The end to end delay between vehicle 0 and vehicle 7 with different protocols is calculated in figure 14. AODV and DumbAgent protocols give the same and the best result compared to DSDV protocol.

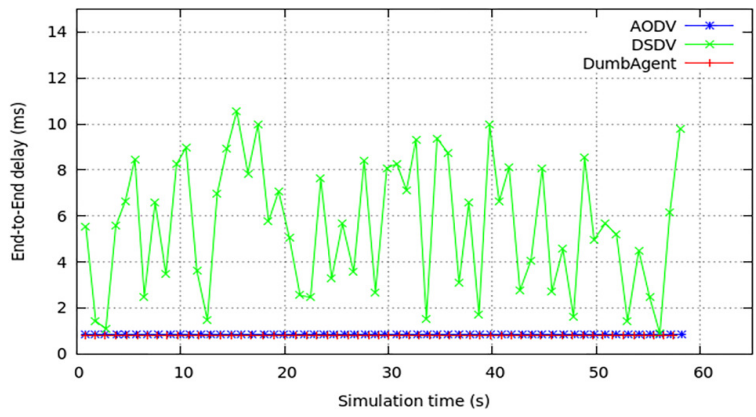

Figure 14. End to end delay between vehicle 0 and vehicle 7 with different protocols (packet size : 500 bytes)

We are moving now to present the average performance of metrics to give more realistic results.

Figure 15 presents the average throughput of all vehicles with different protocols. We can say that the throughput results of the different protocols converge with a slight preference for the DumbAgent protocol. 


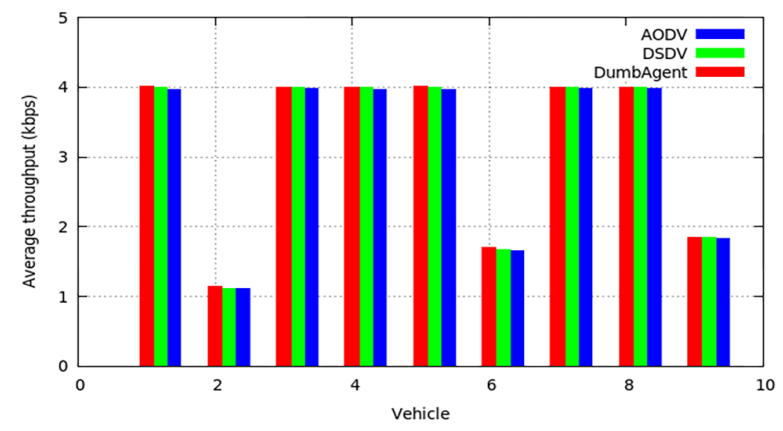

Figure 15. Average throughput of all vehicles with different protocols (packet size: 500 bytes)

Figure 16 presents the average packet loss of all vehicles with different protocols. All protocols present the same results with some preference to DSDV and DumbAgent protocols in vehicle 3, 4 and 6 compared to AODV protocol.

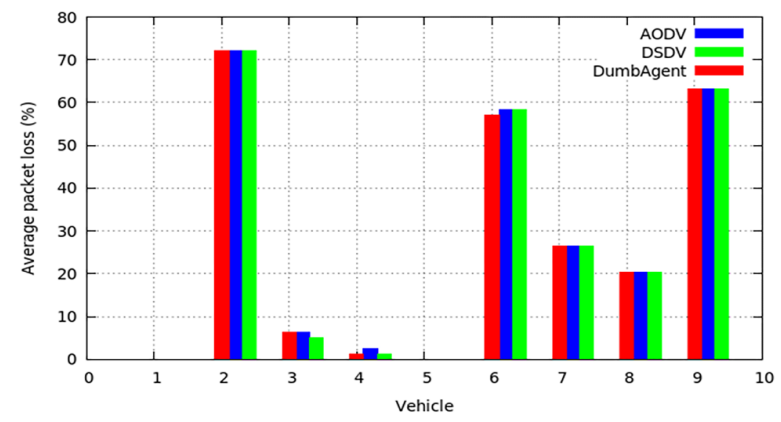

Figure 16. Average packet loss of all vehicles with different protocols (packet size : 500 bytes)

Figure 17 illustrates the average end to end delay between vehicle 0 and other vehicles with different protocols. It is clear that DSDV protocol has the highest value compared to other protocols. AODV and DSDV protocols are the best in term of end to end delay.

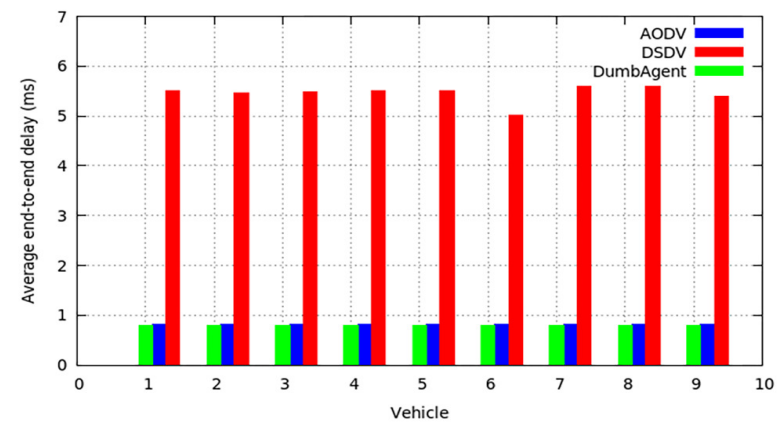

Figure 17. Average end to end delay between vehicle 0 and other vehicles with different protocols (packet size : 500 bytes)

In conclusion, we can say that AODV, DSDV and DumbAgent have the same results in term of throughput and packet loss. AODV and DumbAgent are better than DSDV in term of end to end 
delay. DSDV requests a regular update of its routing tables and makes it gourmand in term of end to end delay.

\section{CONCLUSION}

In this paper, we implemented the standard $802.11 \mathrm{p}$ in NS-2 simulator to evaluate the performance of metrics (Throughput, packet loss and end to end delay) of mobility model graph that is generated by VanetMobisim simulator. The performance of metrics is analyzed according to the variation of packet size, exchanged vehicle speed and routing protocols.

From simulation results we can conclude:

- The throughput, the packet loss and the end to end delay are affected by varying the distance between sender and receiver and no by varying the speed of vehicles.

- When the distance between sender vehicle and receiver vehicles is less than 140 meters, the throughput for all vehicles is the same under the condition that the sending power of the signal, the sensitivity of the receivers and the number of cars in the given physical environment are fixed.

- As a result of varying of the packet size: as the packet size increases, the average throughput and the average end to end delay increases as well. Whenthe value of the packet size s multiplied by $n$ then the throughput value and the end to end delay value are multiplied by $n$ also.

- As a result of varying of the routing protocols: AODV, DSDV and DumbAgent have the same results in term of throughput and packet loss but AODV and DumbAgent are better than DSDV in term of end to end delay since the DSDV is very slow in routing.

As a future, we can extend our work for different radio propagation models and environments [18] [19]. We can also change the mobility model graphs to show his effect in the simulation results.

\section{REFERENCES}

[1] S. Al-Sultan, M.M Al-Doori, A.H. Al-Bayatti and H. Zedan, "A comprehensive survey on vehicular ad hoc network", Journal of Network and Computer Applications, London, UK, pp. 380-392, January 2014.

[2] R. Uzcategui and G. Acosta-Marum, "WAVE: A Tutorial", IEEE Communications Magazine, May 2009.

[3] J. Harri, F. Filali and C. Bonnet, "Mobility Models for Vehicular Ad Hoc Networks: A Survey and Taxonomy”, IEEE Communications Surveys \& Tutorials, Vol. 11, No. 4, pp. 19-41, December 2009.

[4] Y.R.B Al-Mayouf, M. Ismail1 , N. F. Abdullah, S.M. Al-Qaraawi and O.A. Mahdi "Survey on Vanet technologies and simulation models", ARPN Journal of Engineering and Applied Sciences, Vol. 11, No. 15, pp. 9414-9427, August 2016.

[5] F.J. Martinez, C.K. Toh, J.C. Cano, C.T. Calafate and P. Manzoni, "A survey and comparative study of simulators for vehicular ad hoc networks (VANETs)", in Wireless Communications and Mobile Computing, Vol. 11, pp. 813-828, July 2011. 
[6] N.M. Mittal and S. Choudhary, "Comparative Study of Simulators for Vehicular Ad-hoc Networks (VANETs)", International Journal of Emerging Technology and Advanced Engineering, Vol. 4, Issue 4, pp. 528-537, April 2014.

[7] S. Khandelwal, "Comparative Analysis of Network Simulator for Vehicular AdHoc Networks (VANET) Communication", Journal of Advanced Computing and Communication Technologies, Vol. 2, No. 2, April 2014.

[8] "VanetMobiSim", http://vanet.eurecom.fr.

[9] "Network Simulator ns-2", http://www.isi.edu/nsnam/ns/IEEE Wireless Communications, Vol. 13, No. 5, pp. 36-43, 2006.

[10] M. Fiore, J. Haerri, F. Filali and C. Bonnet, "Vehicular Mobility Simulation for VANETs", Proceedings of the 40th Annual Simulation Symposium, pp. 301-309, March 2007.

[11] R.S. Shukla and N. Tyagi, "Performance evaluation of mobility model and routing protocols for inter vehicular communication system", International Conferene on Emerging Trends in Networks and Computer Communications (ETNCC), April 2011.

[12] D. Shree and D. Singh,"Performance Evaluation of Realistic Mobility Models using Road Side Units", International Journal of Computer Applications, Vol. 80, No 15, pp. 0975-8887, October 2013.

[13] Nidhi and D.K. Lobiyal, "Performance Evaluation of VANET Using Realistic Vehicular Mobility", Chapter in Advances in Computer Science and Information Technology, Networks and Communications, Vol. 84 of the series Lecture Notes of the Institute for Computer Sciences, Social Informatics and Telecommunications Engineering pp. 477-489, February 2012.

[14] C.E. Perkins and P. Bhagwat, "Highly dynamic destination-sequenced distance-vector routing (DSDV) for mobile computers", ACM SIGCOMM computer communication review ACM, New York, NY, USA, Vol. 24, pp. 234-244, September 1994.

[15] C.E. Perkins and E.M. Royer, “Ad-hoc On-Demand Distance Vector Routing “, 2nd IEEE workshop on mobile computing systems and applications, pp. 90-100, February 1999.

[16] C.E. Perkins, E.M Belding-Royer and S. Das, "Ad hoc on-demand distance vector (AODV) routing", pp. 1721-2070, July 2003.

[17] M. Treiber, A. Hennecke and D. Helbing, "Congested Traffic States in Empirical Observations and Microscopic Simulations”, Physical Review E, Vol. 62, pp. 1805- 1824. August 2000.

[18] D. Dhoutaut, A. R'egis and F. Spies, "Impact of Radio Propagation Models in Vehicular Ad Hoc Networks Simulations", Proceedings of the 3rd international workshop on Vehicular ad hoc networks, pp. 40-49, September 2006.

[19] R.C. Poonia1 and V.Singh, "PERFORMANCE EVALUATION OF RADIO PROPAGATION MODEL FOR VEHICULAR AD HOC NETWORKS USING VANETMOBISIM AND NS-2”, International Journal of Distributed and Parallel Systems (IJDPS), Vol. 3, No. 4, July 2012. 